\title{
Efficacy of Consolidation High-Dose Chemotherapy with Ifosfamide, Carboplatin and Etoposide (HD-ICE) Followed by Autologous Peripheral Blood Stem Cell Rescue in Chemosensitive Patients with Metastatic Soft Tissue Sarcomas
}

\author{
Marcus Schlemmer ${ }^{a} \quad$ Clemens-Martin Wendtner ${ }^{\mathrm{a}} \quad$ Martin Falk $^{\mathrm{a}}$ \\ Sultan Abdel-Rahman ${ }^{a}$ Thomas Licht ${ }^{c}$ Jens Baumert ${ }^{d}$ Christian Straka ${ }^{b}$ \\ Marcus Hentrich $^{f}$ Christoph Salat ${ }^{\mathrm{a}}$ Wolfgang Hiddemann ${ }^{\mathrm{a}}$ Rolf-Dieter Issels ${ }^{\mathrm{a}} \mathrm{e}^{\mathrm{e}}$

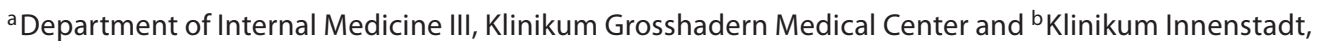 \\ Ludwig Maximilian University, 'Department of Internal Medicine III, Technical University of Munich, \\ ${ }^{\mathrm{d}}$ GSF Institute of Epidemiology, ${ }^{e}$ KKG Hyperthermia, GSF National Research Center for Environment and Health, and \\ ${ }^{\mathrm{f}}$ Hospital Harlaching, Munich, Germany
}

\section{Key Words}

High-dose chemotherapy $\cdot$ Metastasis $\cdot$ Soft tissue sarcoma

\begin{abstract}
Background: Prognosis of patients with metastatic soft tissue sarcomas (MSTS) is poor even after response to doxorubicin-based chemotherapy. We report phase II data of highdose chemotherapy and peripheral blood stem cell (PBSC) rescue in patients with MSTS responding to Al-G chemotherapy. Patients and Methods: From 1997 to 2002, 55 patients with MSTS were prospectively treated with 4 cycles of Al-G (doxorubicin $75 \mathrm{mg} / \mathrm{m}^{2}$, ifosfamide $6 \mathrm{~g} / \mathrm{m}^{2}$ with G-CSF support). Responders received 2 further cycles of AI-G with collection of PBSCs. High-dose chemotherapy consisted of ifosfamide $12 \mathrm{~g} / \mathrm{m}^{2}$, carboplatin $1.2 \mathrm{~g} / \mathrm{m}^{2}$ and etoposide 1.2 $\mathrm{g} / \mathrm{m}^{2}$ (HD-ICE) followed by reinfusion of PBSCs. Results: Twenty-one of 55 patients (38\%) were assessed as responders (3 complete response, 18 partial response). All but 2 pa-
\end{abstract}

tients refusing treatment received high-dose chemotherapy with PBSC rescue leading to grade IV hematologic toxicity without severe infections in all patients. No toxic death occurred. After a median follow-up time of 30 months, the median progression-free time was 12 months and survival time was 22 months for the entire group. By intent-totreat analysis the probability of 5 -year progression-free survival was significantly higher for patients allocated to HD-ICE compared to patients receiving second-line chemotherapy after failure of Al-G (14 vs. 3\%; $p=0.003$ ). The estimated 5year overall survival between the 2 groups was different ( $27 \%$ vs. not reached) but did not reach significance ( $p=$ $0.08)$. Conclusion: HD-ICE is feasible and promising in patients with chemosensitive MSTS. A randomized phase III trial is warranted to further define the role of HD-ICE as consolidation treatment in these patients.

Copyright $\odot 2006$ S. Karger AG, Basel

\section{KARGER}

Fax +41613061234 E-Mail karger@karger.ch www.karger.com (c) 2006 S. Karger AG, Basel

0030-2414/06/0712-0032\$23.50/0

Accessible online at:

www.karger.com/ocl
Marcus Schlemmer, MD

Medizinische Klinik und Poliklinik III - Grosshadern

Marchioninistrasse 15, DE-81377 München (Germany)

Tel. +49 897095 4768, Fax +49897095 4776

E-Mail Marcus.Schlemmer@med.uni-muenchen.de 


\section{Introduction}

Patients with metastatic soft tissue sarcomas (MSTS) have a poor prognosis with response rates to doxorubicin-based chemotherapy between 23 and $45 \%$ and a median overall survival (OS) of about 11.7-15 months [1-4]. As single agents, doxorubicin, ifosfamide and dacarbazine were shown to be most active in soft tissue sarcomas (STS) with an overall response rate between 15 and 28\% [5-8]. For the combination of doxorubicin and ifosfamide, dose-dependent activities were shown $[9,10]$. In a phase II trial of the EORTC Soft Tissue and Bone Sarcoma Group using doxorubicin $75 \mathrm{mg} / \mathrm{m}^{2}$ and ifosfamide $5 \mathrm{~g} / \mathrm{m}^{2}$, a response rate of $45 \%$ was reached leading to a median survival of 15 months [1]. This promising result could not be confirmed in a phase III trial with a response rate of only $23 \%$ and a median OS of 13 months [2]. The most recent EORTC trial in MSTS randomizes between doxorubicin $75 \mathrm{mg} / \mathrm{m}^{2}$ and a dose-intensified combination (doxorubicin $75 \mathrm{mg} / \mathrm{m}^{2}$ and ifosfamide $10 \mathrm{~g} / \mathrm{m}^{2}$ ) to answer the question whether combination chemotherapy provides a survival advantage over single-agent doxorubicin. As the use of hematopoietic growth factors allows safe escalation of dose, several regimes have been tested in patients with metastatic disease. These trials included small numbers of patients with a variety of bone sarcomas and STS [11-14]. Furthermore, high-dose chemotherapy with autologous peripheral blood stem cell rescue (PBSCR) was performed in 18 patients with STS receiving doxorubicin and escalated doses of ifosfamide. The overall response rate was $50 \%$, but OS was only 13 months [15]. In another series including 27 patients treated with epirubicin and ifosfamide, the overall response rate was 38\% [16]. A French protocol used etoposide, cisplatin and ifosfamide in 30 patients with MSTS and while observing high renal toxicity, progression-free survival (PFS) was $21 \%$ at 5 years [17]. In order to better define the role of a similar regimen including ifosfamide, carboplatin and etoposide (ICE) with respect to both toxicity and efficacy, we performed a phase II trial in patients with MSTS responding to doxorubicin/ifosfamide induction chemotherapy supported by G-CSF (AI-G).

\section{Patients and Methods}

\section{Patients' Eligibility}

Patients between 18 and 60 years with histologically confirmed chemonaive MSTS of grade II and III with measurable disease were eligible for this phase II trial. Patients were required to have a good performance status (grade $0-2$ according to WHO) and normal organ functions. Baseline eligibility criteria were normal blood counts $(\mathrm{WBC}>4,000 / \mu \mathrm{l}$, platelet count $>100,000 / \mu \mathrm{l}$ ) and adequate renal (serum creatinine $<1.5 \mathrm{mg} / \mathrm{dl}$ ) and liver (serum bilirubin $<1.5$ $\mathrm{mg} / \mathrm{dl}$ ) function tests. Patients with a history of active cardiac disease, including myocardial infarction and congestive heart disease, were excluded from the study. Further exclusion criteria included histology of malignant mesothelioma, nonmesenchymal chondrosarcoma, neuroblastoma, chordoma, Kaposi's sarcoma and gastrointestinal stromal tumor, as well as a history of another malignancy, central nervous system involvement and prior radiotherapy on parameter lesions. The study was performed according to the guidelines established by the Helsinki Declaration. The protocol was approved by the Ethics Committee of the Ludwig Maximilian University, Germany (No. 93/97). Written informed consent was obtained from all patients enrolled onto this study.

\section{Treatment}

Induction chemotherapy consisted of doxorubicin $75 \mathrm{mg} / \mathrm{m}^{2}$ as $30 \mathrm{~min}$ infusion on day 1 and ifosfamide $1.5 \mathrm{~g} / \mathrm{m}^{2}$ as $120 \mathrm{~min}$ infusion on days 1-4 (AI-G). Twenty-four hours after the end of chemotherapy, G-CSF was given subcutaneously at a dose of $5 \mu \mathrm{g} / \mathrm{kg}$ body weight/day until neutrophil counts exceeded 3,000/ $\mu$ l. AI-G cycles were repeated on day 22 . After 4 cycles, responding patients received 2 further cycles of AI-G chemotherapy with an increased dose of G-CSF ( $10 \mu \mathrm{g} / \mathrm{kg}$ body weight/day). If more than $10 \mathrm{CD} 34+$ cells/ $\mu \mathrm{l}$ were found in peripheral blood, leukapheresis was performed to harvest at least $2 \times 10^{6} \mathrm{CD} 34+$ cells/kg body weight for autologous stem cell transplantation. The interval between the end of induction therapy or surgery and the start of high-dose chemotherapy was 4 weeks according to protocol. Highdose chemotherapy consisted of ifosfamide $2 \mathrm{~g} / \mathrm{m}^{2}$ ( $1 \mathrm{~h}$ infusion) from day -8 until day -3 , carboplatin $200 \mathrm{mg} / \mathrm{m}^{2}$ ( $1 \mathrm{~h}$ infusion) from day -8 until day -3 and etoposide $200 \mathrm{mg} / \mathrm{m}^{2}$ ( $22 \mathrm{~h}$ infusion) from day -8 until day -3 (HD-ICE). This resulted in total doses of $12 \mathrm{~g} / \mathrm{m}^{2}$ ifosfamide, $1,200 \mathrm{mg} / \mathrm{m}^{2}$ carboplatin and $1,200 \mathrm{mg} / \mathrm{m}^{2}$ etoposide. After 2 days without chemotherapy, patients received at least $2 \times 10^{6} \mathrm{CD} 34+$ cells $/ \mathrm{kg}$ body weight via central venous catheter on day 0 . G-CSF ( $5 \mu \mathrm{g} / \mathrm{kg}$ body weight) was given subcutaneously from day 1 until recovery of neutrophils.

\section{Staging Procedures and Treatment Evaluation}

At enrollment, physical examination, full laboratory analysis of blood parameters, creatinine clearance and cardiac ultrasound were performed. Additionally, contrast-enhanced CT scan of chest and abdomen and, if needed, MRI of the site of disease were performed. During induction treatment, blood counts, physical examination and toxicity assessment were performed after each cycle of AI-G chemotherapy. After 4 cycles, all patients were evaluated for response according to WHO criteria by contrast-enhanced CT scans, MRI, or both, based on the extent of the disease defined at presentation [18]. Two independent investigators reviewed the files and films of all responding patients. Toxicity was evaluated according to the Common Toxicity Criteria [19]. Before the start of high-dose chemotherapy, possible infections were excluded and serological blood tests were taken. During follow-up, staging was repeated every 3 months in the first year and every 6 months thereafter.

The duration of response was measured from the start of induction treatment to the date of documented progression; the du- 
Table 1. Patients' characteristics

\begin{tabular}{lc}
\hline Characteristics & Patients \\
\hline Sex & \\
$\quad$ Male & 34 \\
$\quad$ Female & 21 \\
Age at entry & \\
19-40 years & 26 \\
$\quad 41-60$ years & 29 \\
Site of metastases & \\
$\quad$ Lung & 24 \\
Lung and liver & 7 \\
$\quad$ Lung and lymph node & 4 \\
Lung, liver and bone & 2 \\
Liver & 4 \\
Liver and bone & 2 \\
Lymph node & 8 \\
Bone & 4 \\
Prior treatment & \\
$\quad$ None & 16 \\
Surgery alone & 24 \\
Surgery plus radiation & 15 \\
Radiation alone & 0 \\
\hline
\end{tabular}

Table 2. Histologic diagnoses and grades

\begin{tabular}{llrrr}
\hline \multirow{2}{*}{\begin{tabular}{l} 
Cell type \\
\cline { 2 - 5 }
\end{tabular}} & Patients & & \\
\cline { 2 - 5 } & grade 2 & grade 3 & total & $\%$ \\
\hline Leiomyosarcoma & 6 & 10 & 16 & 29 \\
Liposarcoma & 3 & 1 & 4 & 7 \\
Rhabdomyosarcoma & 0 & 3 & 3 & 5 \\
PNET & 1 & 2 & 3 & 5 \\
MFH & 2 & 1 & 3 & 5 \\
Synovial sarcoma & 1 & 4 & 5 & 10 \\
Hemangiopericytoma & 3 & 2 & 5 & 10 \\
Other & 4 & 12 & 16 & 29 \\
\hline
\end{tabular}

PNET $=$ Primitive neuroectodermal tumor MFH = malignant fibrous histiocytoma.

ration of complete response (CR) was calculated from the moment CR was documented to the first evidence of progression. PFS was measured from the date of treatment start to documented progression. Survival was calculated from the time of treatment start to the date of death.

The primary endpoint was PFS. Secondary endpoints were response to induction chemotherapy and OS.

\section{Statistics}

OS and PFS were estimated according to the method of Kaplan and Meier [20]. The 95\% confidence intervals (CIs) of the KaplanMeier estimates were calculated with Greenwood's variances [21].
The endpoints for actuarial analysis were PFS and OS. The comparison of survival parameters in responding versus nonresponding patients was performed using the log-rank test [22]. For all tests, $\mathrm{p}<0.05$ was considered to be statistically significant.

This protocol was designed as multicenter, nonrandomized, controlled single arm phase II study with PFS as primary endpoint. Sample size estimation for patients with MSTS was performed for the improvement in PFS from 12 to 24 months by the log-rank test. The estimated response rate was 34\% (23-45\%). In case of 5.5 years of recruitment and 5 -year follow-up, the required number was estimated as 53 patients ( $\alpha=5 \%, \beta=20 \%)$.

\section{Results}

\section{Patients' Characteristics}

Between April 1997 and December 2002, 55 patients with MSTS were treated according to the protocol. The study population consisted of 34 male and 21 female patients with a median age of 40.5 years (range 18.8-60.5 years). Details are shown in tables 1,2 .

\section{Feasibility and Toxicity of Induction Chemotherapy $(A I-G)$}

Forty-six patients (84\%) received 4 induction chemotherapy cycles (AI-G) according to the study protocol. One patient refused further treatment after 1 cycle, 6 patients progressed after 2 cycles and 2 patients after $3 \mathrm{cy}$ cles. The median number of induction chemotherapy cycles given was 4 (range 1-4). Before high-dose chemotherapy, 21 patients were found eligible for PBSCR, 2 patients refused PBSCR.

During induction chemotherapy, nonhematologic toxicity was mainly mild, while no severe side effects (grade 3 and 4) were seen. The most frequent side effects were alopecia (grade 2) which was observed in all patients, and nausea (grade 1) which was seen in the majority of patients (70\%). Hematologic toxicity was significant in terms of leukopenia (30\% grade 3 and $15 \%$ grade 4) and thrombocytopenia which occurred in almost every patient during the induction treatment, but did not differ from previously published data for doxorubicin/ifosfamide chemotherapy combined with GCSF [1].

\section{Response to Induction Therapy (AI-G), Stem Cell Collection and Surgery}

All patients were evaluated after 4 cycles of AI-G chemotherapy. An objective response was observed in 21 (38\%) of the 55 patients, including $3 \mathrm{CR}$ and 18 partial responses (PR). No change was observed in 20 patients 
(37\%) and 14 (25\%) progressed during induction therapy (table 3). All 21 patients with chemosensitive tumors received 2 additional cycles of AI-G followed by peripheral blood stem cell apheresis. Collection of peripheral blood stem cells was achieved for these patients with a harvest efficiency of at least $2 \times 10^{6} \mathrm{CD} 34+$ cells/kg body weight. During mobilization chemotherapy, none of the patients progressed. Before high-dose chemotherapy (HD-ICE), 4 patients underwent surgery. One patient had radical (R0) resection of his lung metastases. In 2 patients R0 surgery of their primary tumor could be obtained, and in $1 \mathrm{pa}-$ tient R1 resection of the primary tumor was achieved. Before the start of HD-ICE, 6 patients had no evidence of disease, 8 patients had only distant disease with no evidence of disease at the primary tumor site and 7 patients entered high-dose chemotherapy with persistent disease at local and distant sites.

\section{Toxicity and Feasibility of High-Dose Chemotherapy}

(HD-ICE)

Of 21 patients responding to AI-G (3 CR and $18 \mathrm{PR}$ ), 19 patients received high-dose chemotherapy with PBSCR while 34 patients were not eligible for high-dose chemotherapy. Two patients being eligible for HD-ICE refused this treatment. HD-ICE as conditioning regimen was feasible and no death due to therapy occurred. The median number of infused mononuclear CD34+ cells was $6 \times$ $10^{6} / \mathrm{kg}$ body weight (range $2-12 \times 10^{6}$ ). After HD-ICE chemotherapy, all patients experienced grade 3 or 4 neutropenia and thrombocytopenia (table 4). The maximum anemia seen in the trial was of grade 3 , as patients received packed red cells before the hemoglobin decreased below $7.5 \mathrm{~g} / \mathrm{dl}$. The median number of transfused packed red cell units during high-dose chemotherapy was 2 (range 0-4). The median duration of grade 4 neutropenia and thrombocytopenia was 9.3 (7-14) and 6.9 days (3-17), respectively. Due to severe thrombocytopenia (grade 4), 11 patients required a median of 2 platelet transfusions (range $1-5)$. Four patients suffered from severe pneumonia, which resolved after combined antibiotic treatment. Other infectious complications were mild, only fever of unknown origin with a median duration of 3.5 days (0-12) was observed. Mucositis was severe (grade 3) in 6 patients requiring infusion of morphine. Hepatotoxicity with elevation of bilirubin $>3 \times \mathrm{N}$, most likely due to drug toxicity, was observed in 1 patient on day 1 after PBSCR, resolving spontaneously. At a median of 13 days after PBSCR, patients were discharged from hospital. Up to now, with a median follow-up of 30 months, no late toxicity like myelodysplasia, leukemia or solid tumors were seen.

High-Dose Chemotherapy in

Chemosensitive STS
Table 3. Radiographic response to induction chemotherapy

\begin{tabular}{lcr}
\hline Radiographic response & Patients & $\%$ \\
\hline CR & 3 & 6 \\
PR & 18 & 32 \\
No change & 20 & 37 \\
Progressive disease & 14 & 25 \\
\hline Total & 55 & 100
\end{tabular}

Table 4. Maximal toxicity during high-dose chemotherapy (HDICE; $\mathrm{n}=18$ )

\begin{tabular}{|c|c|c|c|c|c|}
\hline & \multicolumn{5}{|c|}{ Patients with CTC grade toxicity } \\
\hline & 0 & 1 & 2 & 3 & 4 \\
\hline Leukopenia & 0 & 0 & 0 & 3 & 15 \\
\hline Neutropenia & 0 & 0 & 0 & 1 & 17 \\
\hline Thrombopenia & 0 & 0 & 0 & 3 & 15 \\
\hline Anemia & 0 & 0 & 5 & 13 & 0 \\
\hline Infection & 3 & 1 & 8 & 6 & 0 \\
\hline Mucositis & 1 & 3 & 8 & 6 & 0 \\
\hline Nausea & 3 & 0 & 10 & 5 & 0 \\
\hline Nephrotoxicity & 14 & 4 & 0 & 0 & 0 \\
\hline Hepatotoxicity & 5 & 9 & 3 & 1 & 0 \\
\hline Neurotoxicity & 17 & 0 & 1 & 0 & 0 \\
\hline Cardiotoxicity & 18 & 0 & 0 & 0 & 0 \\
\hline
\end{tabular}

One patient refused further therapy after day 1 of HD-ICE. This patient was excluded from the toxicity evaluation. CTC = Common Toxicity Criteria.

\section{Relapse and Survival}

After a median follow-up time of 30 months, the median time to progression for the entire study group of 55 patients was 12 months (95\% CI 8-15). The 2- and 5-year PFS rate estimated according to Kaplan-Meier was 18\% (95\% CI 8-28) and 7\% (95\% CI 0-8), respectively (fig. 1). In an intent-to-treat analysis including all 21 patients responding to AI-G induction therapy, the 2-year PFS for patients allocated to HD-ICE $(\mathrm{n}=21)$ was $32 \%(95 \% \mathrm{CI}$ $12-52$ ) compared to $9 \%$ (95\% CI 0-18) in patients being treated with second-line chemotherapy after failure of AI-G induction ( $p=0.0029)$. The median PFS for patients in the high-dose group was 16 months in contrast to the conventional treatment arm with a median PFS of only 7 months (fig. 2). The difference in probability of 5-year PFS in the high-dose group $(\mathrm{n}=21)$ with $14 \%(95 \%$ CI $5-47)$ compared to the standard treatment arm $(n=34)$ 


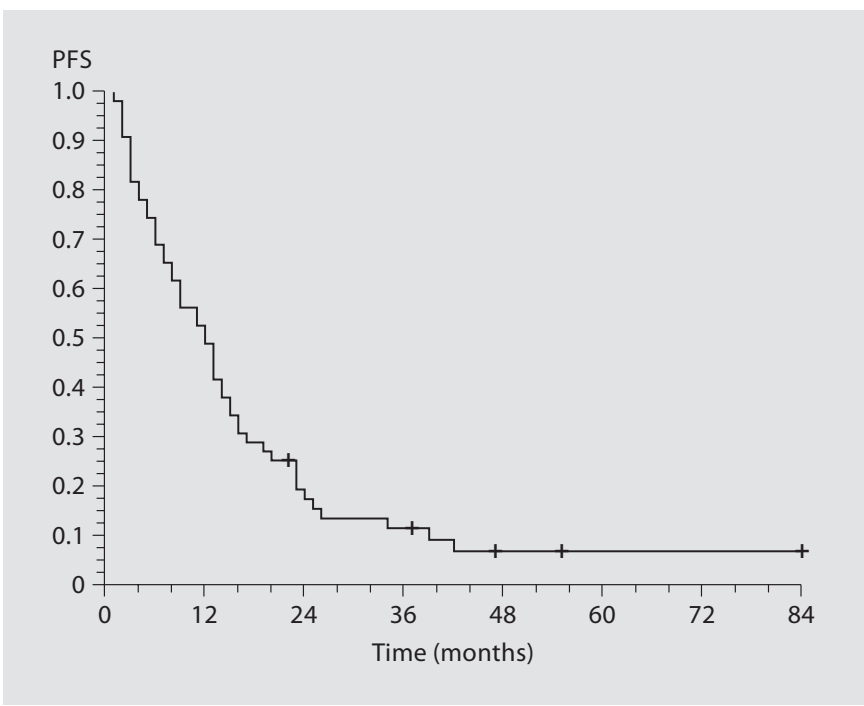

Fig. 1. PFS in the entire cohort of 55 patients.

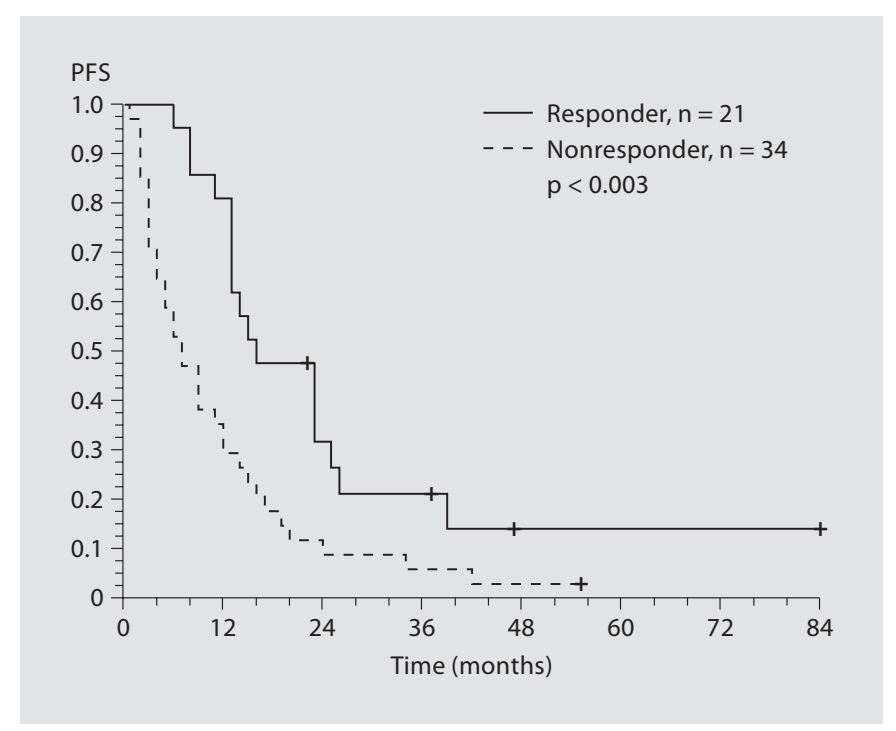

Fig. 2. $P F S$ in patients responding to $A I-G$ induction and being allocated to HD-ICE $(n=21)$ versus patients receiving conventional second-line chemotherapy after failure to induction treatment (no HD-ICE; $\mathrm{n}=34$; intent-to-treat analysis).

with $3 \%$ (95\% CI 0-9) was statistically significant $(\mathrm{p}=$ 0.003). One patient with neurofibrosarcoma and lung metastases being in PR after induction chemotherapy refusing HD-ICE died after 39 months due to progression of the lung metastasis, another being in CR after induction therapy and also refusing HD-ICE, died 13 months

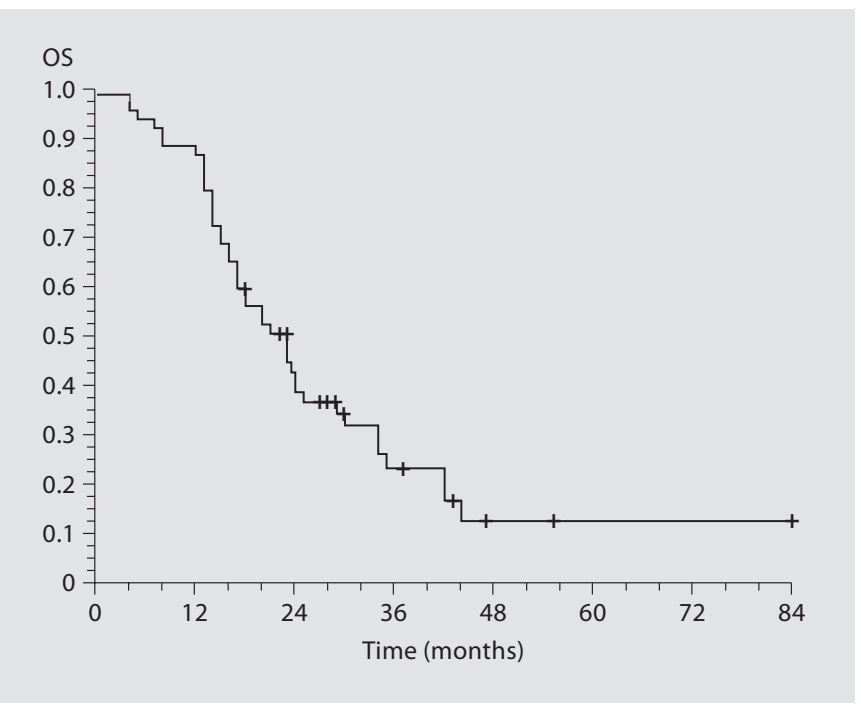

Fig. 3. OS in the entire cohort of 55 patients.

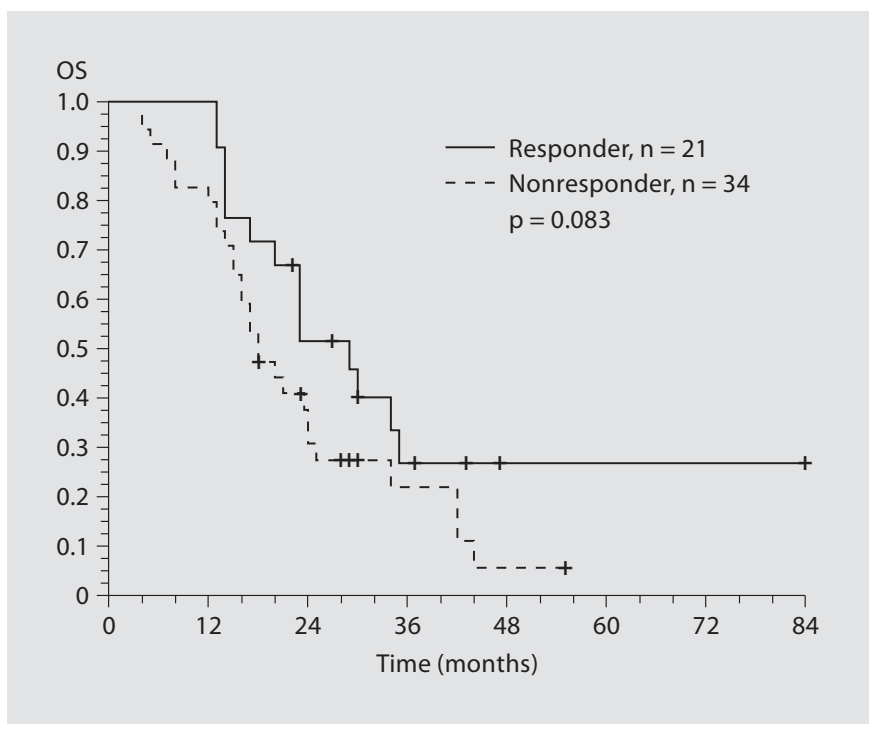

Fig. 4. OS in patients responding to AI-G induction and being allocated to HD-ICE $(n=21)$ versus patients receiving conventional second-line chemotherapy after failure to induction treatment (no HD-ICE; $\mathrm{n}=34$; intent-to-treat analysis).

after the start of the study due to progressive disease. At present, 7 of the 21 responding patients are alive. The 5year estimated OS rate of all patients is $12 \%$ with a median OS time of 22 months (fig. 3). The probability of OS was not higher for patients responding (CR, $P R)$ to induction chemotherapy compared to nonresponders. In an in- 
tent-to-treat analysis, the median OS for the patients being allocated to high-dose chemotherapy $(\mathrm{n}=21)$ was 24 months versus 18 months for nonresponders to AI-G induction. There was no statistical difference in OS between patients with stable and progressive disease $(\mathrm{p}=$ $0.14)$. The estimated 4 -year OS rate was $27 \%$ (95\% CI $7-$ 47) for the high-dose group and 5\% (95\% CI 0-11) for the conventional treatment arm. Estimated 5-year OS in the 2 groups did not reach the level of significance $(\mathrm{p}=0.08$; fig. 4).

\section{Discussion}

The objective of the present study was to investigate efficacy in terms of OS and PFS after HD-ICE and PBSCR given as consolidation therapy in chemosensitive patients with MSTS. Therefore, HD-ICE was tested in a well-defined subgroup of patients experiencing CR or PR to AI-G chemotherapy. The design of our study is similar to a previously conducted study in 30 patients with advanced STS receiving high-dose chemotherapy consisting of ifosfamide, etoposide and cisplatin (VIC) [17]. The authors reported renal toxicity of grade 1 in $43 \%$ and grade 2 or higher in $20 \%$ of the patients using the VIC regimen. Replacing cisplatin by carboplatin in our HDICE regimen was favorable with no grade 2 nephrotoxicity observed. The hematologic toxicity of the HD-ICE regime was comparable to the VIC protocol in terms of duration of aplasia and number of required transfusions.

The OS rate in our present series at 2 and 5 years is 40 and $12 \%$, respectively, compared to the reported OS and PFS rates at 5 years after high-dose chemotherapy based on the VIC regimen, i.e. 23 and $21 \%$, respectively. In addition, the percentage of patients with CR before highdose chemotherapy was higher in the former series (27\%) compared to our study population (6\%). This is of major importance since the percentage of long-term survivors among patients with metastastic or advanced STS achieving a CR after conventional chemotherapy is a predictor of survival rates in a given series of patients $[4,12,17,23]$. In a large series of the EORTC Soft Tissue and Bone Sarcoma Group including 2,185 patients with defined firstline chemotherapy, a performance status of 0 , female gender and grade I histology were independent parameters correlating to 5 -year survival besides achievement of a CR after first-line chemotherapy [24]. In our study, grade I tumors were excluded by definition of high-risk criteria while most of the patients presented at an initial perfor- mance status of 1 . Furthermore, the majority of our patients were male. Also, the absence of liver metastases was shown to be associated with longer survival times in a multivariate analysis [24]. Our study comprised 12 patients with hepatic metastases explaining an adverse survival outcome.

The CR rate of $6 \%$ after AI-G induction was low and in our study 7 patients are alive, 3 without evidence of disease at $37+, 47+$ and $84+$ months, and 4 patients with disease at $22+, 30+, 37+$ and $43+$ months. One patient who did not qualify for high-dose chemotherapy due to lack of response to AI-G chemotherapy is alive. This patient was formally diagnosed as having a gastrointestinal stromal sarcoma which has meanwhile been reclassified as a gastrointestinal stromal tumor and is now responding to treatment with imatinib.

The present study was negative for its primary endpoint, i.e. PFS could not be prolonged from 12 to 24 months. For the entire study population PFS was only 12 months in accordance with previous reports and patients in the high-dose group showed only a slight improvement in PFS with 16 months. Comparing patients having received high-dose chemotherapy with those having received best second-line chemotherapy - although this non-randomized phase II study was not powered to answer this question sufficiently - reveals that OS does not seem to be improved by high-dose chemotherapy. Only PFS, which is a valid endpoint for phase II studies [25] was better for those patients receiving high-dose chemotherapy. It has to be stressed that it remains to be demonstrated in a phase III trial whether high-dose chemotherapy is able to improve survival of those patients who are in CR before high-dose chemotherapy. High-dose therapy has been evaluated more extensively in pediatric patients with Ewing's sarcomas, rhabdomyosarcomas and osteosarcomas. Also, in this patient group several studies conclude that high-dose chemotherapy does not significantly improve treatment outcome compared to conventional relapse therapy $[26,27]$. Transplantation-related side effects and the threat of secondary malignant neoplasm after high-dose chemotherapy may complicate the future course of patients who are currently viewed as progression-free survivors [28]. Histological subtypes which might benefit from dose-intensified chemotherapy should be selected for high-dose chemotherapy strategies. Patients with peripheral primitive neuroectodermal tumors were found to have a superior PFS to patients with other histological sarcoma subtypes [29]. The concept of tandem high-dose chemotherapy, established for other tumor entities, should also be further studied for STS after 
first pilot studies have proved the feasibility of this approach in sarcomas $[30,31]$. Our data raise the question whether high-dose chemotherapy and PBSCR or the intensified dose of ifosfamide $\left(12 \mathrm{~g} / \mathrm{m}^{2}\right)$ in the ICE regime is responsible for the long-term survival of these patients. As a large number of phase II trials showed only limited activity of several new agents in STS [32,33], a possible approach is to increase the CR rate to conventional chemotherapy by escalating the dose of drugs active against STS. The dosage of ifosfamide in our AI-G regimen was suboptimal with $6 \mathrm{~g} / \mathrm{m}^{2}$. Meanwhile numerous trials have suggested a dose-response relationship for ifosfamide [2, 34]. In a previous trial it was shown that multiple cycles of dose-intensive therapy with high-dose ifosfamide can be administered safely, resulting in CR rates above $20 \%$ [15]. It is an open question whether the dose intensity of ifosfamide in our HD-ICE regimen alone warrants an advantage for patients with metastatic STS. We therefore plan a randomized phase III trial (high-dose ifosfamide vs. HD-ICE) with a larger sample size, to answer this question for patients with chemosensitive MSTS.

\section{References}

1 Steward WP, Verweij J, Somers R, et al: Granulocyte-macrophage colony-stimulating factor allows safe escalation of dose-intensity of chemotherapy in metastatic adult soft tissue sarcomas: a study of the European Organization for Research and Treatment of Cancer Soft Tissue and Bone Sarcoma Group. J Clin Oncol 1993;11:15-21.

2 Le Cesne A, Judson I, Crowther D, et al: Randomized phase III study comparing conventional-dose doxorubicin plus ifosfamide versus high-dose doxorubicin plus ifosfamide plus recombinant human granulocyte-macrophage colony-stimulating factor in advanced soft tissue sarcomas: a trial of the European Organization for Research and Treatment of Cancer/Soft Tissue and Bone Sarcoma Group. J Clin Oncol 2000;18:26762684.

3 Verweij J, Lee SM, Ruka W, et al: Randomized phase II study of docetaxel versus doxorubicin in first- and second-line chemotherapy for locally advanced or metastatic soft tissue sarcomas in adults: a study of the European Organization for Research and Treatment of Cancer Soft Tissue and Bone Sarcoma Group. J Clin Oncol 2000;18:2081-2086.

4 Blay JY, van Glabbeke M, Verweij J, et al: Advanced soft-tissue sarcoma: a disease that is potentially curable for a subset of patients treated with chemotherapy. Eur J Cancer 2003;39:64-69.

5 Buesa JM, Mouridsen HT, van Oosterom AT, et al: High-dose DTIC in advanced soft-tissue sarcomas in the adult: a phase II study of the EORTC Soft Tissue and Bone Sarcoma Group. Ann Oncol 1991;2:307-309.

6 Edmonson JH, Ryan LM, Blum RG, et al: Randomized comparison of doxorubicin alone versus ifosfamide plus doxorubicin or mitomycin, doxorubicin, and cisplatin against advanced soft tissue sarcomas. J Clin Oncol 1993;11:1269-1275.
7 Elias AD, Eder JP, Skea T, et al: High-dose ifosfamide with mesna uroprotection: a phase I study. J Clin Oncol 1990;8:170-178.

8 Nielsen OS, Judson I, van Hoesel Q, et al: Effect of high-dose ifosfamide in advanced soft tissue sarcomas: a multicentre phase II study of the EORTC Soft Tissue and Bone Sarcoma Group. Eur J Cancer 2000;36:61-67.

9 O’Bryan RM, Baker LH, Gottlieb JB, et al: Dose response evaluation of adriamycin in human neoplasia. Cancer 1997;39:19401948.

10 Patel SR, Vadhan-Raj S, Burgess MA, et al Results of two consecutive trials of dose-intensive chemotherapy with doxorubicin and ifosfamide in patients with sarcomas. Am J Clin Oncol 1998;21:317-321.

11 Elias AD, Ayash LJ, Wheeler C, et al: Phase I study of high-dose ifosfamide, carboplatin and etoposide with autologous hematopoietic stem cell support. Bone Marrow Transplant 1995;15:373-379.

12 Dumontet C, Biron P, Bouffet E, et al: High dose chemotherapy with ABMT in soft tissue sarcomas: a report of 22 cases. Bone Marrow Transplant 1992;10:405-408.

13 Kessinger A, Petersen K, Bishop M, et al: High dose therapy with autologous hematopoietic stem cell rescue for patients with metastatic sarcoma. Proc Am Soc Clin Oncol 1994; $13: 1674$.

14 Kasper B, Lehnert T, Bernd L, et al: Highdose chemotherapy with autologous peripheral blood stem cell transplantation for bone and soft-tissue sarcomas. Bone Marrow Transplant 2004;34:37-41.

15 Bokemeyer C, Franzke A, Hartmann JT, et al: A phase I/II study of sequential, dose-escalated, high-dose ifosfamide plus doxorubicin with peripheral blood stem cell support for the treatment of patients with advanced soft tissue sarcomas. Cancer 1997;80:12211227.
16 Reichardt P, Tilgner J, Hohenberger P, Dorken B: Dose-intensive chemotherapy with ifosfamide, epirubicin, and filgrastim for adult patients with metastatic or locally advanced soft tissue sarcoma: a phase II study. J Clin Oncol 1998;16:1438-1443.

17 Blay JY, Bouhour D, Ray-Coquard I, et al: High-dose chemotherapy with autologous hematopoietic stem-cell transplantation for advanced soft tissue sarcoma in adults. J Clin Oncol 2000;18:3643-3650.

18 Miller AB, Hoogstraten B, Staquet M, et al: Reporting results of cancer treatments. Cancer 1981;47:207-214.

19 Trotti A, Byhardt R, Stetz J, et al: Common Toxicity Criteria - version 2.0: an improved reference for grading the acute effects of cancer treatment - impact on radiotherapy. Int J Radiat Oncol Biol Phys 2000;47:13-47.

20 Kaplan E, Meier P: Nonparametric estimation from incomplete observations. J Am Stat Assoc 1958;53:457-481.

21 Greenwood M: The Natural Duration of Cancer: Reports on Public Health and Medical Subjects. London, Her Majesty's Stationery Office, 1926, vol 33.

22 Anderson JR, Cain KC, Gelber RD: Analysis of survival by tumor response. J Clin Oncol 1983;1:710-719.

23 Boulad F, Kernan NA, LaQuaglia MP, et al: High-dose induction chemoradiotherapy followed by autologous bone marrow transplantation as consolidation therapy in rhabdomyosarcoma, extraosseous Ewing's sarcoma, and undifferentiated sarcoma. J Clin Oncol 1998;16:1697-1706.

24 Van Glabbeke M, van Oosterom AT, Oosterhuis JW, et al: Prognostic factors for the outcome of chemotherapy in advanced soft tissue sarcoma: an analysis of 2,185 patients treated with anthracycline-containing firstline regimens - a European Organization for Research and Treatment of Cancer Soft Tissue and Bone Sarcoma Group study. J Clin Oncol 1999;17:150-157. 
25 Van Glabbeke M, Verweij J, Judson I, Nielsen OS; EORTC Soft Tissue and Bone Sarcoma Group: Progression-free rate as the principal end-point for phase II trials in soft-tissue sarcomas. Eur J Cancer 2002;38:543-549.

26 Weigel BJ, Breitfeld PP, Hawkins D, Crist WM, Baker KS: Role of high-dose chemotherapy with hematopoietic stem cell rescue in the treatment of metastatic or recurrent rhabdomyosarcoma. J Pediatr Hematol Oncol 2001;23:272-276.

27 Sauerbrey A, Bielack S, Kempf-Bielack B, Zoubek A, Paulussen M, Zintl F: High-dose chemotherapy (HDC) and autologous hematopoietic stem cell transplantation (ASCT) as salvage therapy for relapsed osteosarcoma. Bone Marrow Transplant 2001;27:933937.
28 Burdach S, van Kaick B, Laws HJ, et al: Allogeneic and autologous stem-cell transplantation in advanced Ewing tumors: an update after long-term follow-up from two centers of the European Intergroup study EICESS. Stem-Cell Transplant Programs at Dusseldorf University Medical Center, Germany and St. Anna Kinderspital, Vienna, Austria. Ann Oncol 2000;11:1451-1462.

29 Bertuzzi A, Castagna L, Nozza A, Quagliuolo V, Siracusano L, Balzarotti M, Compasso S, Alloisio M, Soto Parra H, Santoro A: Highdose chemotherapy in poor-prognosis adult small round-cell tumors: clinical and molecular results from a prospective study. J Clin Oncol 2002;20:2181-2188.

30 Kozuka T, Kiura K, Katayama H, et al: Tandem high-dose chemotherapy supported by autologous peripheral blood stem cell transplantation for recurrent soft tissue sarcoma. Anticancer Res 2002;22:2939-2944.
31 Faglioli F, Aglietta M, Tienghi A, et al: Highdose chemotherapy in the treatment of relapsed osteosarcoma: an Italian sarcoma group study. J Clin Oncol 2002;20:21502156.

32 Van Oosterom AT, Verweij J: New drugs for the treatment of sarcomas. Hematol Oncol Clin North Am 1995;9:909-925.

33 Okuno S, Ryan LM, Edmonson JH, et al: Phase II trial of gemcitabine in patients with advanced sarcomas (E1797): a trial of the Eastern Cooperative Oncology Group. Cancer 2003;97:1969-1973.

34 Elias AD: High-dose therapy for adult soft tissue sarcoma: dose response and survival. Semin Oncol 1998;25(suppl 4):19-23. 